\title{
The use of PET in evaluating patients with primary brain tumours: is it useful?
}

\author{
William C Olivero, Scott C Dulebohn, J Richard Lister
}

\begin{abstract}
During an 18 month period 39 patients were evaluated with $\left[{ }^{18} \mathrm{~F}\right]$ fluorodeoxyglucose-PET (FDG-PET) for primary brain tumours. These included patients with suspected newly diagnosed tumours and patients with known tumours who were being evaluated for possible recurrence or increasing tumour grade. Scans were performed on a 951-31 Siemen's PET scanner with $4 \mathrm{~mm}$ resolution. Scanning time was about 20 minutes per patient. All patients had undergone recent cerebral MRI. These patients were divided into two groups. In the first group (30) MRI and PET concurred on the diagnosis. The second group (nine) comprised those where the interpretation of MRI and PET was different or there was a question of the diagnosis on MRI. This group comprised three patients in whom MRI suggested recurrent tumour and PET inaccurately suggested radiation necrosis; two patients with newly diagnosed enhancing lesions on MRI in whom PET was useful in distinguishing strokes from tumour; two patients with prior gliomas with new enhancing isolated lesions on MRI in whom PET scan accurately depicted radiation necrosis; and two patients with newly diagnosed enhancing lesions on MRI in whom PET scan was helpful in distinguishing multiple sclerosis from tumour in one but not in the other. Therefore, of the 39 patients, PET was helpful in five in distinguishing tumour from other disease processes; but, in so far as influencing treatment, it seemed helpful in only two. Thus PET seems to be of limited value as an aid to evaluating and treating patients with suspected or known primary brain tumours.
\end{abstract}

(F Neurol Neurosurg Psychiatry 1995;58:250-252)

Keywords: glioma; PET; magnetic resonance imaging

With the advent of PET, it is possible to perform functional analysis of different areas of the brain and of brain tumours. Higher grade gliomas were found to have higher metabolic activity on $\left[{ }^{18} \mathrm{~F}\right]$ fluorodeoxyglu-
cose-PET (FDG-PET) than lower grade tumours and radiation necrosis. ${ }^{12}$ False negative and false positive results have, however, been reported. ${ }^{3-5}$

Magnetic resonance imaging with and without gadolinium has been used extensively in the study of primary brain tumours. Usually low grade tumours have a characteristic appearance on MRI and show little or no enhancement as opposed to high grade tumours, which are almost always enhanced on contrast. The agreement between MRI and PET for distinguishing low grade from high grade tumours is considered to be high, ${ }^{3}$ but PET has been considered superior to $M R I$ in distinguishing radiation necrosis from recurrent or residual tumour or increase in tumour grade. ${ }^{23}$

Although PET can give information not available through other imaging modalities, it is difficult to know what the clinical utility of PET is in managing patients with primary brain tumours. Thus, we retrospectively analysed 39 patients with known or presumed primary brain gliomas to determine when the results of PET changed or influenced the clinical course or treatment of these patients. We also compared the accuracy of PET and the neuroradiologist's interpretation of concurrent MRI.

\section{Methods}

Between June 1991 and December 1992, 39 patients had FDG-PET performed for follow up of known primary brain tumours ( 35 patients) or newly suspected tumour (four patients). The PET scans were performed on a 951-31 Siemen's PET scanner, which has $4 \mathrm{~mm}$ resolution. Scanning time was about 20 minutes per patient. The PET scans were read by a radiologist with special training in their interpretation. Magnetic resonance imaging performed on a 1.5 Tesla unit with $\mathrm{T} 1$, $\mathrm{T} 2$, and proton weighted images and gadolinium enhanced T1 weighted images, usually within two weeks of the PET. The office and hospital records of all the patients were reviewed by an independent evaluator to determine the accuracy of PET and when PET seemed to influence the clinical outcome or the treatment of the patients.

\section{Results}

We divided the patients into two groups. In 
the first group (30 patients) were those in whom MRI and PET concurred on the diagnosis. These included nine patients with low grade tumours where the MRI was interpreted as low grade glioma, postoperative change, or recurrent low grade glioma and PET was hypometabolic in the region of the tumour. These patients have all had stable clinical courses or have been reoperated on and had confirmed low grade tumours. Twenty patients have had MRI interpreted as recurrent or residual high grade tumour or increase in tumour grade, and these patients have shown hypermetabolism on PET. One patient with a high grade glioma has had MRI and PET that showed no residual tumour six years after operation. Although PET offered confirmatory data in these patients, it did not seem to alter the clinical course or treatment regimen.

Most patients with recurrent high grade tumour or increase in tumour grade died fairly soon after this diagnosis was made (average six months). The decision to reoperate was made on whether the recurrence was in an accessible area, was causing mass effect, and on the preoperative Karnofsky score. The PET results did not seem to influence that decision. As far as influencing the decision to give chemotherapy, most of the young patients were already on some chemotherapeutic regimen or, especially in elderly patients, it was considered that chemotherapy would not be helpful.

In nine patients, the interpretations of MRI and PET were different or there was a question of the diagnosis on MRI. Three patients with known primary gliomas, all previously operated on and given radiotherapy, had a deterioration in clinical state and an MRI consistent with recurrent high grade tumour (or increase in tumour grade). The PET scan was thought to be consistent with radiation necrosis. Two of these three had high grade tumours confirmed by biopsy. One had a subsequent PET that showed hypermetabolism, consistent with a recurrent tumour.

Two patients with previous surgery and radiotherapy for glioma had MRI with contrast enhancing lesions without significant mass effect. A PET scan showed hypometabolism in these regions, indicative of radiation necrosis. One patient has been stable and the other has had a slow clinical deterioration over several years, so although helpful, PET did not influence treatment.

MRI and PET findings

\begin{tabular}{|c|c|c|c|c|}
\hline & \multicolumn{2}{|l|}{$M R I$} & \multicolumn{2}{|l|}{ PET } \\
\hline & Enhancing & Non-enhancing & Hypometabolic & Hypermetabolic \\
\hline High grade glioma & 23 & 0 & 3 & 20 \\
\hline Low grade glioma & 0 & 9 & 9 & 0 \\
\hline Radiation necrosis & 2 & 0 & 2 & 0 \\
\hline Multiple sclerosis & 2 & 0 & 1 & 1 \\
\hline Stroke & 2 & 0 & 2 & 0 \\
\hline $\begin{array}{l}\text { No residual } \\
\text { High grade tumour }\end{array}$ & 0 & 1 & $\overrightarrow{1}$ & 0 \\
\hline
\end{tabular}

Values are numbers of patients.
There were four patients with newly diagnosed enhancing lesions on MRI in whom PET was done because of the clinical suspicion that these lesions were not tumours although tumour was the differential diagnosis on MRI. Two patients, both with biopsy proved multiple sclerosis, had PET. The follow up postoperative scan of one patient showed that the enhancing lesion had increased in size. This patient had had a previous craniotomy for a presumed glioma. Consideration was given to rebiopsy because of the possibility that the first biopsy was in error, but PET showed a hypometabolic lesion. With increased steroid dosage the lesion has essentially resolved, and the patient has been stable clinically. The other patient had an enhancing lesion on MRI and hypermetabolism on PET suggestive of a tumour. A stereotaxic biopsy showed demyelinating disease and the patient has improved with steroid medication. In one of these patients, the results of PET were useful in obviating the need for rebiopsy.

Two patients with the clinical presentation of stroke had enhancing lesions on MRI; PET showed hypometabolism and the clinical course and subsequent MRI have been consistent with stroke. In one of these patients, with a thalamic lesion, stereotaxic biopsy was considered but PET obviated the need for this. Therefore, it was considered that PET was helpful in five patients (one with multiple sclerosis, two with radiation necrosis, two with stroke) in distinguishing tumour from other disease processes. In so far as influencing treatment, however, it seemed useful in one patient with multiple sclerosis and one patient with a thalamic stroke. Table 1 summarises the PET and MRI findings.

\section{Discussion}

The usefulness of a diagnostic test depends on its accuracy, whether other available tests give the same or better information, and whether the information obtained will influence treatment decisions or the ultimate prognosis of the patient. Data on the accuracy of PET in brain gliomas is still forthcoming. In the original paper by DiChiro et $a l$, all 10 patients with high grade astrocytomas showed a region of high activity and a visible hot spot and none of the 13 patients with low grade gliomas showed this. All cases were, however, not proved by biopsy. Others have reported varying degrees of accuracy of PET in differentiating high grade from low grade tumours and high grade tumours from radiation necrosis. ${ }^{3}$ Valk et al studied the use of PET in malignant cerebral tumours after interstitial brachytherapy. ${ }^{5}$ The overall accuracy of PET for differentiating tumour recurrence from absence of active tumour was $85 \%$. Sensitivity for detection of recurrence was $88 \%$ and specificity $81 \%$. Of note, histological examination of the resected tissues showed apparently viable tumour in all of the cases regardless of the eventual clinical outcome or PET results. Our experience is 
similar to others in that most patients with high grade gliomas show hypermetabolism on PET and those with presumed radiation necrosis and low grade tumours show hypometabolism, but false negative and false positive results are possible.

Because PET does not have the spatial resolution to give adequate anatomical information with regard to tumour and important structures of the brain, it is usually done in conjunction with CT or MRI. Davis et al compared FDG-PET with gadolinium enhanced MRI imaging of neoplasms of the CNS. ${ }^{3}$ Most lesions showed both enhancement with gadolinium and hypermetabolism with FDG-PET and were primarily high grade gliomas. Also, three lesions were hypometabolic on FDG-PET, had no gadolinium enhancement, and were well differentiated astrocytomas. Some anaplastic tumours were, however, hypometabolic on PET and benign tumours were hypermetabolic. Similarly, some anaplastic tumours did not show enhancement on MRI. Our experience is similar in that MRI and PET are usually fairly accurate in predicting the underlying histology but inaccuracies can occur with both techniques.

The work up and treatment of suspected or known primary brain gliomas remains controversial. Low grade gliomas may have long clinical courses without any treatment; high grade gliomas have a poor prognosis no matter what is done. The utility of new treatment regimens such as brachytherapy, radiosurgery, and chemotherapy in most instances remains to be proved. ${ }^{67}$ Also, the role of reoperation for recurrent high grade tumours is unclear. From the medical literature, it does not seem that PET results influence the decision to reoperate on patients. In the report of Valk et al on the use of PET in malignant cerebral tumours after interstitial brachytherapy, nine of 19 patients with positive PET underwent reoperation and nine of 16 patients with negative PET underwent reoperation. ${ }^{5}$ In the study of Janus et al on PET in patients with primary malignant brain tumours, 13 of 20 patients that underwent reoperation had positive scans and seven had negative scans. ${ }^{4}$ These same authors, however, considered that in patients evaluated by FDG-PET but not with biopsy, the addition of the PET information seemed to help the clinician in evaluating the patient. This was especially true in those patients who had had conventional radiotherapy. In 24 of 30 patients further treatment was based on this information-that is, no change with a negative scan and change in chemotherapy with a positive scan. It is not clear from their data, however, whether this change in chemotherapy made any significant difference in the patient's outcome. Kornblith and Walker concluded that at present, there are no chemotherapeutic agents available with sufficient potency or specificity that are capable of producing a therapeutically useful response in a sufficient percentage of patients with malignant gliomas. ${ }^{7}$ We believe that the role of any new potential prognostic indicator such as PET in patients with primary malignant brain tumours is likely to be small until new treatments that can radically change clinical outcomes become available.

In summary, we studied 39 patients with known or suspected primary brain tumours by MRI and PET. In most patients (30/39), PET and MRI concurred on the diagnosis. Also, in most patients (37/39), the results of PET did not seem to influence the treatment or outcome. We found PET useful when MRI showed an enhancing lesion with primary glioma in the differential diagnosis but the clinical history suggested another disease process. In those cases when PET showed hypometabolism, it helped support the decision to defer biopsy and follow up the patient clinically.

We gratefully acknowledge the secretarial assistance of Mrs JoAnna Gass in the preparation of the manuscript.

1 DiChiro G, DeLaPaz RL, Brooks RA, et al. Glucose utilization of cerebral gliomas measured by $\left.{ }^{18} \mathrm{~F}\right]$ fluorodeoxyglucose and positron emission tomography. deoxyglucose and positron

2 DiChiro G, Oldfield E, Wright DC, et al. Cerebral necrosis after radiotherapy and/or intraarterial chemotherapy for brain tumours: PET and neuropathologic studies. AfR Am f Radiol 1988;150:189-97.

3 Davis WK, Boyko OB, Hoffman JM, et al. $\left[{ }^{18} \mathrm{~F}\right] 2$-fluoro-2deoxyglucose-positron emission tomography correlation of gadolinium-enhanced MR imaging of central nervous

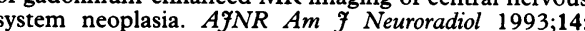
515-23.

4 Janus TJ, Kim EE, Tilbury $\mathrm{R}$, et al. Use of $\left[{ }^{18} \mathrm{~F}\right]$ fluorodeoxyglucose positron emission tomography in patients with primary malignant brain tumours. Ann Neurol 1993;33:540-8.

5 Valk PE, Budinger TF, Levin VA, et al. PET of malignant cerebral tumours after interstitial brachytherapy. Demonstration of metabolic activity and correlatic
with clinical outcome. $\mathcal{F}$ Neurosurg 1988;19:830-8.

6 Gutin PH, Leibel SA, Wara WM, et al. Recurrent malig nant gliomas: survival following interstitial brachytherapy with high-activity iodine-125 sources f Neurosurg 1987;67:864-73.

7 Kornblith PL, Walker M. Chemotherapy for malignant gliomas. $\mathcal{f}$ Neurosurg 1988;68:1-17. 